\title{
NUMERICAL STUDY OF THE INTERACTION BETWEEN FOCUSED ULTRASOUND WAVE AND BRAIN-LIKE SOFT MATERIAL
}

\author{
ZHIBO DU ${ }^{1}$, ZHANLI LIU ${ }^{2}$ \\ ${ }^{1}$ Department of Engineering Mechanics, Tsinghua University \\ Beijing 100084, China \\ dzb17@mails.tsinghua.edu.cn \\ ${ }^{2}$ Department of Engineering Mechanics, Tsinghua University \\ Beijing 100084, China \\ liuzhanli@tsinghua.edu.cn
}

Key words: Focused ultrasound, Brain-like soft material, Multi-field coupled simulation.

\begin{abstract}
Focused ultrasound is of great significance in the fields of medical ultrasound imaging, diagnosis, and treatment, yet little is known about the quantification of the physical effects of focused ultrasound and the analysis of the corresponding biological effects. In this paper, the acoustic-solid-thermal coupled computational model is developed to study the interaction between focused ultrasound and brain-like soft materials. Firstly, a hyperviscoelastic constitutive model is established to describe the dynamic and thermal-mechanical behaviour of brain-like soft material. Due to the high compression resistance and low shear resistance of brain-like soft materials, it will appear in the focus area that Compression strain and shear strain are approximate, but compressive stress is much larger than shear stress. Secondly, the dynamic mechanical response of brain-like soft materials under focused ultrasound excitation with different frequencies and amplitudes were studied. By analyzing the hysteresis loop of the feature points in the focus area, it can be concluded that the loss angle of dynamic modulus of brain-like soft materials increases with the increase of load frequency and the increase of load frequency. The higher the load amplitude is, the greater the energy input to the focusing region is, and the faster the dissipation speed is. At last, through the multi-field coupled simulation, it is found that the focusing accuracy of the focused ultrasound increases with the increase of the frequency, but the focusing energy intensity will decrease. The input energy has an optimized value in a specific frequency range. The research is helpful for the improvement of focused ultrasound diagnosis, imaging and treatment technology, and the use of dynamic mechanical property inversion of brain-like soft materials.
\end{abstract}

\section{INTRODUCTION}

Because of its non-ionizing radiation, low cost, real-time imaging, high resolution, and portability, ultrasound is widely used in hospitals and clinics to diagnose various diseases. Especially for obstetrics and cardiology, ultrasound is the best choice for diagnosis ${ }^{[1]}$. In the process of medical ultrasound diagnosis, to obtain better longitudinal resolution and better echo quality, the ultrasound is usually converged to form focused ultrasound. Ultrasound can be applied to medical treatment by focusing the ultrasound energy in a highly localized way. 
Focused ultrasound can penetrate the skin and tissue layer, and act on the deep target position of the human body in a non-invasive way. The target tissue was ablated or intervened by mechanical loading, cavitation force or heating to achieve the therapeutic effect, which can ensure the necessary strength on the target tissue without damaging the surrounding tissue. Focused ultrasound has been used in tumor ablation, blood clot dissolution and ultrasound enhanced drug delivery ${ }^{[2]}$. Tumor ablation is achieved by absorbing the energy of the focused beam and generating a lot of heat. Blood clot dissolution and ultrasound-enhanced drug delivery are performed by the introduction of ultrasound contrast agents to induce cavitation ${ }^{[3,4]}$.

In conclusion, there are a lot of applications of focused ultrasound in the diagnosis, imaging, and treatment of medical ultrasound, but there are still some problems in the use of focused ultrasound. For example, for the sake of safety, the ultrasonic metrology commonly used in medical ultrasound imaging is generally less than the average sound intensity of $10^{-1} \mathrm{~W} / \mathrm{cm}^{2}$, but the average sound intensity of ultrasound will rise significantly during the use of focused ultrasound, especially for the application of focused ultrasound in the treatment process. During the treatment, the average sound intensity of the focus area can reach $10^{2}-10^{4} \mathrm{~W} / \mathrm{cm}^{2}$. At this time, in addition to the effect of ultrasound on biological tissues at the target, healthy biological tissues may also be damaged. It is not clear about the quantification of the physical effect of focused ultrasound and the mechanism of its biological effect. Therefore, further characterization of the process of focused ultrasound is needed to promote the clinical application of focused ultrasound further. Numerical simulation is a powerful method for analyzing focused ultrasound It can obtain the fields of sound, force, and heat during the focusing process, which is condreive to analyzing the force-thermal response law during focusing process.

Modeling for focuse the target of focused ult biological soft tissue is challenging to perfo mechanical characterization under dynamic loading. For example, at a strain rate of $1000 \mathrm{~s}-1$,

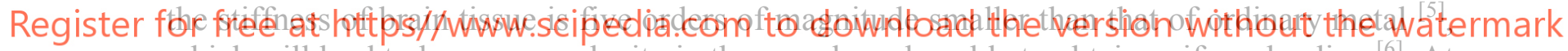
which will lead to low wave velocity in the sample and unable to obtain uniform loading ${ }^{[6]}$. At the same time, the low intensity of the brain tissue leads to the need for more accurate mechanical signal measurement methods ${ }^{[7]}$, and the low intensity makes it difficult to distinguish the inherent mechanical response from the inertial effect in high-speed experiments ${ }^{[8,9]}$. However, all brain tissues showed considerable sensitivity to the applied strain rate.

Therefore, this paper abstracts and simplifies the interaction between focused ultrasound and brain-like soft materials in the medical field, and establishes an acoustic-solid-thermal coupling model for studying the interaction between focused ultrasound and brain-like soft materials. By establishing the hyper-viscoelastic constitutive equation to describe the dynamic mechanical behavior of brain-like soft materials, the corresponding model is used to study the influence of ultrasonic frequency on the attenuation of ultrasonic energy and the accuracy of focused ultrasound. The dynamic mechanical response characteristics of brain-like soft materials under different frequency and amplitude loads are further studied.

\section{ACOUSTIC-SOLID-THERMAL COUPLED COMPUTATIONAL MODEL}

This section describes the problem of focused ultrasound from the transducer acting on 
biological tissues through the water. An axisymmetric acoustic-solid-thermal coupling model was established. The hyperviscoelastic constitutive model is used to describe the nonlinear elasticity and broadband response characteristics of brain-like soft materials. The detailed information such as the boundary, contact method, mesh division and element type in the multiphysics coupling model are introduced.

\subsection{Establishment of acoustic-solid-thermal coupling model}

In this paper, the process of the interaction between focused ultrasound and brain-like soft materials is simplified as the model shown in figure 1A, that is, the bowl-shaped acoustic transducer and biological tissue exist in the water together. The bowl-shaped acoustic transducer transforms the electrical signal into the focused ultrasonic signal which propagates to the focus. The focused ultrasound propagates to the biological tissue through the water and focuses in a specific area to form a focus area. The central opening of the bowl-shaped acoustic transducer is $10 \mathrm{~mm}$, and the focal length is $63.64 \mathrm{~mm}$; the biological tissue height is $75.5 \mathrm{~mm}$, and the radius is $48.6 \mathrm{~mm}$.

The above process is simulated and reproduced, and the finite element model is shown in figure $1 \mathrm{~B}$ is established. The model is established in an axisymmetric manner. The bowl-shaped acoustic transducer itself is omitted in the calculation, and a water area is established at the position of the bowl-shaped acoustic transducer, and the shape of the water area is matched with the bowl-shaped acoustic transducer. A layer of transitional waters is established between
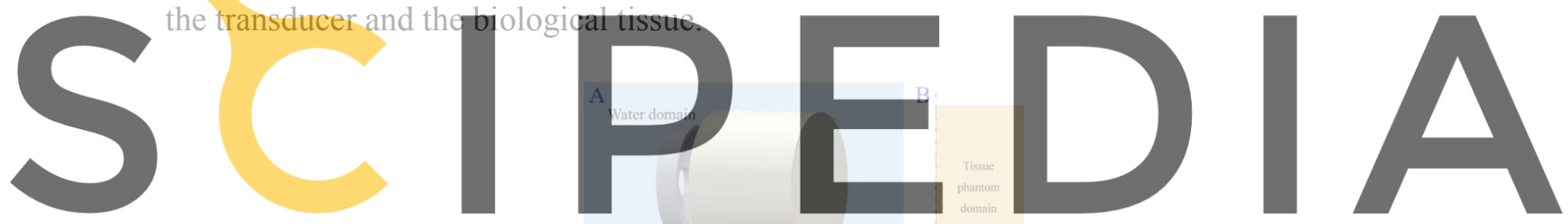

Register for free at https//www.scipedia.com to download the version without the watermark

Figure 1: A. Simplified model; B. finite element model

For the heat conduction process, a simplified Pennes equation is used:

$$
\rho C_{P} \frac{\partial T}{\partial t}=\nabla \cdot(k \nabla T)+Q
$$

where $T$ is the temperature, and $\rho$ is the density, and $C_{P}$ is the specific heat, and $k$ is the thermal conductivity, and $Q$ is the unit time unit volume heat production ${ }^{[10]}$. The corresponding parameters are shown in Table 1.

In the acoustic-solid-thermal coupling mode, the stress value is calculated from the strain or displacement by using the finite element equation, and the sound intensity is calculated according to the stress. The value of the heat source term of each integral point is calculated from the sound intensity, which is brought into the biological heat conduction equation. The temperature value at each point is obtained, and finally brought into the thermal expansion equation, so that the influence of heat on displacement or strain is calculated, and finally, a complete cycle is formed as shown in the figure 2. 


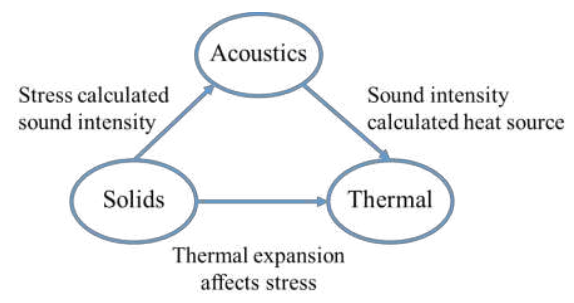

Figure 2: Acoustic-solid-thermal coupling method

\subsection{Numerical calculation model building method}

In this paper, a hyper-viscoelastic constitutive model is used, and material parameters obtained by experimental fitting at different frequencies are used to ensure that the loading at different frequencies can be simulated accurately.

The hyperelastic part adopts the Mooney-Rivlin model, and the strain energy function is a polynomial function with principal strain invariant:

$$
U=C_{10}\left(\bar{I}_{1}-3\right)+C_{01}\left(\bar{I}_{2}-3\right)+\left(J_{e l}-1\right)^{2} / D_{1}
$$

where $C_{10} C_{01}, D_{1}$ is the material parameter, and the steady shear modulus $G_{0}=2\left(C_{10}+C_{01}\right)$ and the bulk modulus $k_{0}=2 / D_{1}$; wherein, $\bar{I}_{1}$ and $\bar{I}_{2}$ are the first and second invariants of the principal strain, and $J_{e l}=J / J_{\text {th }}$ are related to the third invariants of the principat strain, and
$J_{t h}=\left(1+\varepsilon_{t h}\right)^{3}$ is related to the linear thermal expansion. All hyperelastic parameters are shown
in Table 1.
The viscous part adopts the Miaxwell viscoelastic model, and the Cauchy stress exprossion
is as follows:

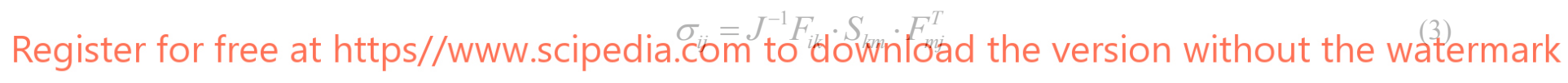
where $\sigma_{i j}$ is Cauchy stress, and $F$ is deformation gradient tensor, and $S_{i j}$ is the second Piola Kirchhoff stress, which can be calculated by genetic integration:

$$
S_{i j}=\int_{0}^{t}\left[G_{i j k l}(t-\tau) \partial E_{k l} / \partial \tau\right] d \tau
$$

where $E_{k l}$ is the green strain component and $G_{i j k l}$ is stress relaxation function. For isotropic materials, the relaxation modulus can be expressed in the form of the Prony series:

$$
G(t)=G_{\infty}+\sum_{i=1}^{n} G_{i} e^{-t / \tau_{i}}
$$

where $G_{\infty}$ is the long-term modulus, and $\tau_{i}$ is relaxation time. The relaxation modulus and relaxation time refer to the results in the literature ${ }^{[11]}$, which is fitted with the experimental data in the literature (brain material test is provided in the range of $0.01 \mathrm{~Hz}-10 \mathrm{MHz}{ }^{[12-18]}$. All viscoelastic parameters are shown in Table 1.

For the water area, the Tait equation is used to describe the change of fluid pressure: 


$$
P=B\left[\left(\rho / \rho_{0}\right)^{\Gamma_{0}+1}-1\right]
$$

where $B$ and $\Gamma_{0}$ are constants. For the water area, the value of $\Gamma_{0}$ is 6.15 , the bulk modulus is $2.19 \mathrm{GPa}$, and the value of $B$ is obtained by the following formula:

$$
K=B\left(\Gamma_{0}+1\right)
$$

At the same time, a small shear modulus is assigned to the water area to ensure the stability of the numerical calculation ${ }^{[19]}$, and the corresponding parameters are shown in Table 1.

Table 1: Material parameters

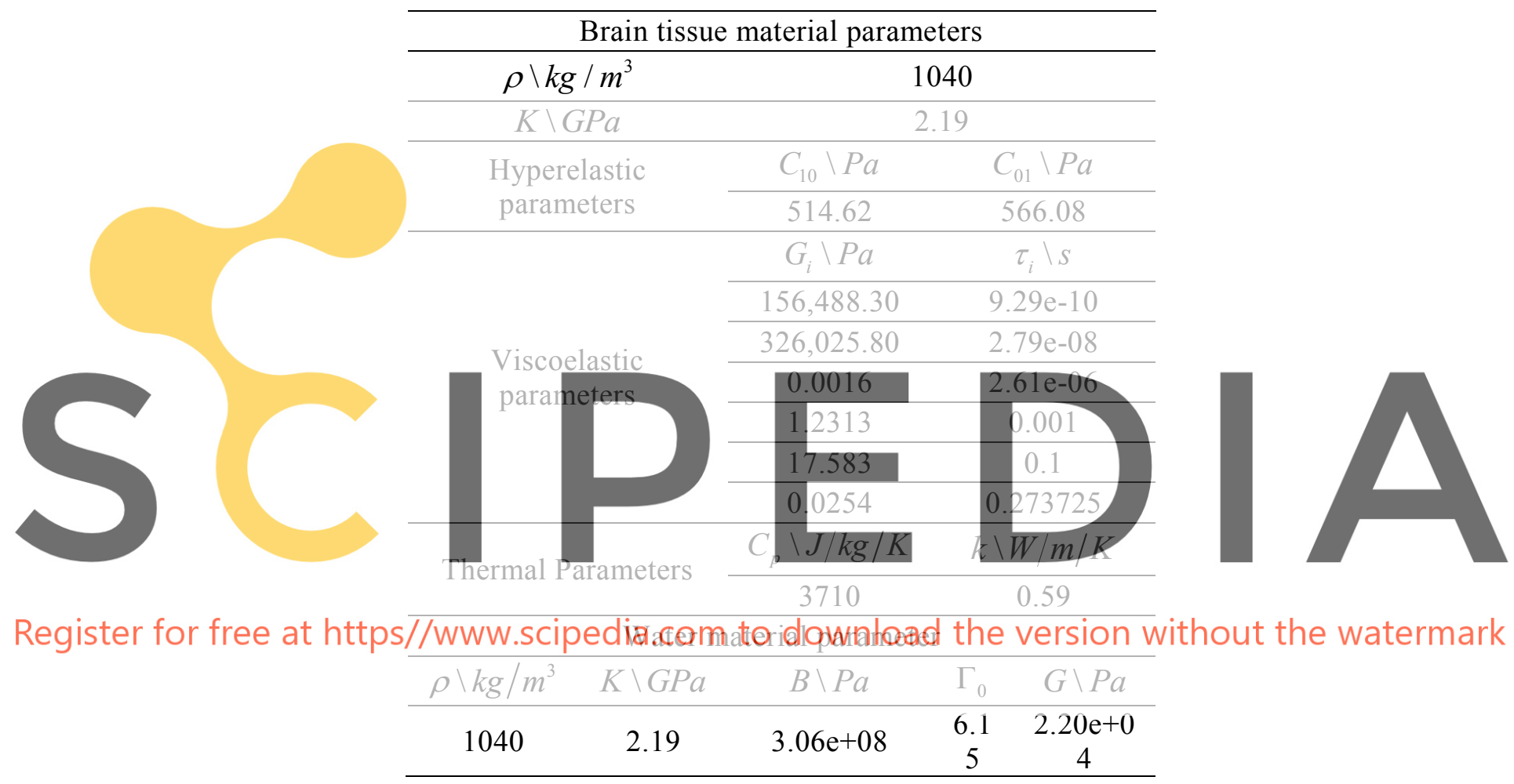

For the finite element model, as shown in figure 1B, the loading mode is selected as follows: during the simulation, the pressure load is applied at the arc of the bowl-shaped acoustic transducer. The selected contact mode is: the transducer water area, transitional water area, and biological tissue area are connected by Tie mode in the simulation process. The free boundary is adopted as the boundary condition because the time course of the loading process is in millisecond order, the whole displacement of the model can be ignored, so the displacement of the rigid body need not be restricted mainly. For the finite element mesh density, the mesh density is determined according to the input load, and the corresponding wavelength is calculated according to the wave speed and frequency:

$$
\lambda=c / f
$$

In the process of calculation, at least ten grids are required within a wavelength range, that 
is, the grid size is needed to be less than one-tenth of the wavelength. The entire calculation process uses a dynamic temperature-displacement coupling explicit calculation method.

\section{DISCUSSION AND RESULTS}

In this section, the acoustic-solid-thermal coupling model is used to describe the propagation characteristics of focused ultrasound in brain-like soft material, and to explain the special stressstrain response of brain-like soft material due to the high compression resistance and low shear resistance. The dynamic mechanical behavior and energy dissipation characteristics of the focus area and its internal characteristic points are analyzed by using the hysteresis effect and temperature rise rate.

\subsection{Loading form with $1 \mathrm{MHz}$ frequency and $1 \mathrm{MPa}$ amplitude}

First, an ultrasonic signal with a load frequency of $1 \mathrm{MHz}$ and a pressure amplitude of 1 $\mathrm{MPa}$ is applied as an example to observe the situation during the focusing process on an acoustic solid thermal coupling model. According to the observation of the compression wave, as shown in figure 3, the compression wave is emitted from the bowl-shaped transducer, and the beam gradually converges to the symmetry axis through the water area and brain like soft material area. During the focusing process, the amplitude of compressed wave in the water area increases gradually, and the amplitude of compressed wave in the brain like soft material area decreases gradually due to the energy attenuation. Finally, a stable elliptical focusing area is formed near

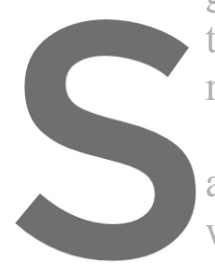
the symmetry axis as resolution in ultrasonic

During the focusing at the interface between wave deflection generat imaging.
process, it can be found
the water area and the
es a secondary shear wa
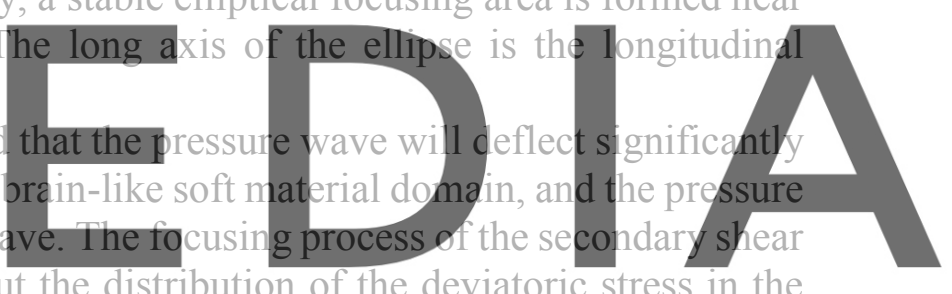

wave and the compression wave is similar, but the distribution of the deviatoric stress in the

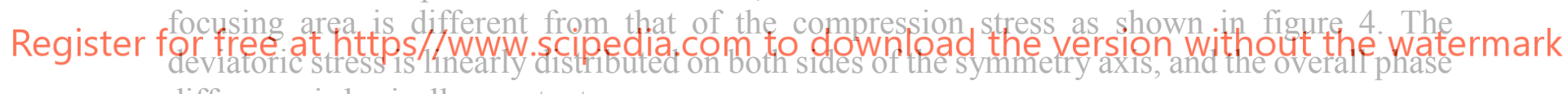

difference is basically constant.

There are both compressional waves and secondary shear waves in the brain-like soft material domain, so the stress state in the brain-like soft material domain is relatively complicated. We can use the Von Mises stress to characterize the complex stress state. The expression of Von Mises stress is as follows:

$$
S=\sqrt{\left[\left(\sigma_{11}-\sigma_{22}\right)^{2}+\left(\sigma_{11}-\sigma_{33}\right)^{2}+\left(\sigma_{22}-\sigma_{33}\right)^{2}+6\left(\sigma_{12}^{2}+\sigma_{13}^{2}+\sigma_{23}^{2}\right)\right] / 2}
$$

where $\sigma_{11}, \sigma_{22}, \sigma_{33}, \sigma_{12}, \sigma_{13}, \sigma_{23}$ are the stress components. As shown in figure 5A, observing the distribution of Von Mises stress, it can be found that the overall stress distribution is basically consistent with the distribution of pressure and shear stress, and the magnitude of the stress amplitude at the focal area and the interface are larger, which is consistent with the shear stress distribution.

Energy attenuation in brain-like soft material domains, which causes temperature changes in the acoustic-solid-thermal coupling model. As shown in figure 5B, observing the temperature distribution at the same time as the Von Mises stress cloud map, it can be found that the 
temperature distribution is basically consistent with the Von Mises stress distribution. The area corresponding to the high-temperature region is the place where the Von Mises stress is higher, and the contour of the temperature rise region is basically consistent with the contour of Von Mises stress.

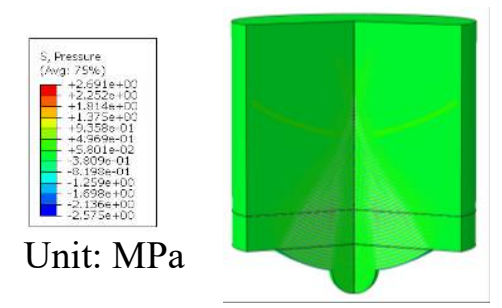

Figure 3: The cloud map of steady-state pressure distribution

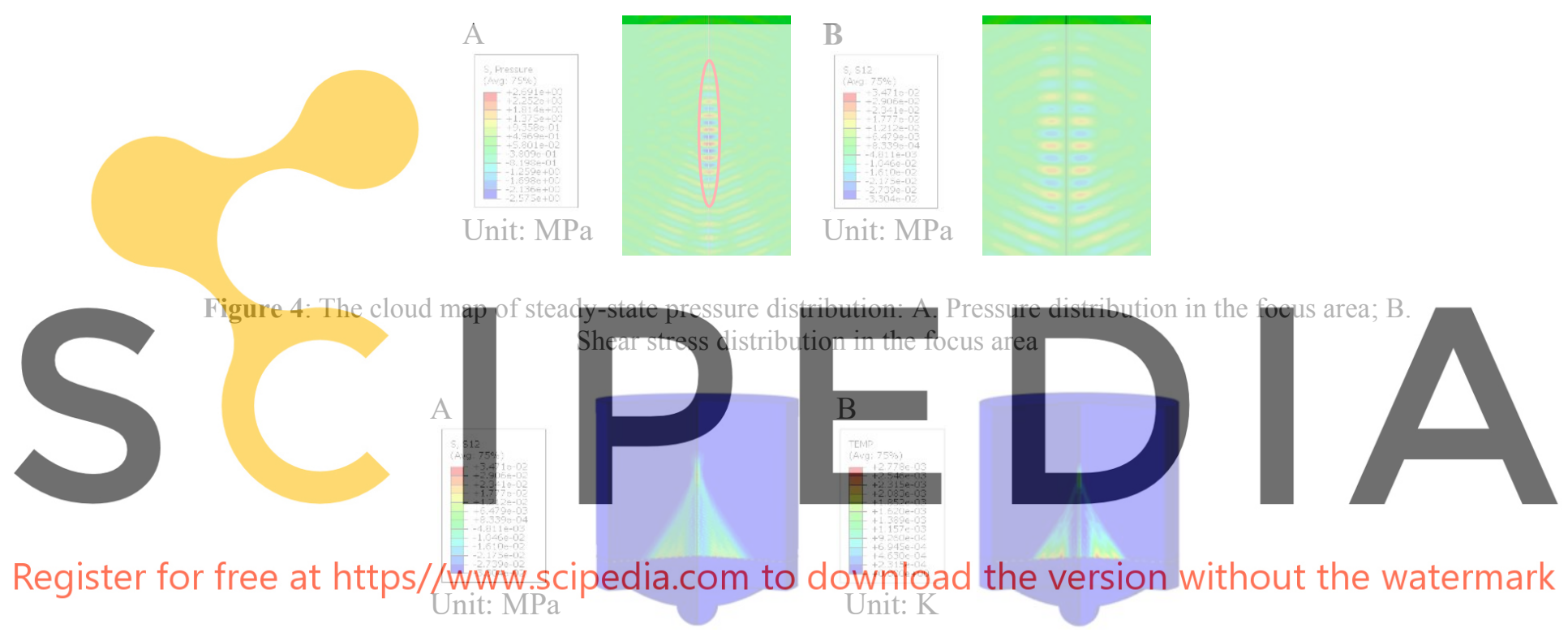

Figure 5: Comparison of the distribution of Von Mises stress and temperature: A. Von Mises stress distribution; B. Temperature distribution

Furthermore, in order to observe the stress-strain response in the focus area, ultrasonic signals with different loads and frequencies are applied to the model. As shown in figure 6, taking the central point of the focus area for observation, it can be found that the strain amplitudes of compression wave and secondary shear wave are close no matter how the load amplitude changes.

In order to observe the change of stress state caused by compression wave and secondary shear wave, the concept of stress triaxiality can be further introduced

$$
P / \sigma_{M}=\sqrt{2}\left(\sigma_{1}+\sigma_{2}+\sigma_{3}\right) / 3 \sqrt{\left(\sigma_{1}-\sigma_{2}\right)^{2}+\left(\sigma_{1}-\sigma_{3}\right)^{2}+\left(\sigma_{2}-\sigma_{3}\right)^{2}}
$$

which is the ratio of pressure to Mises stress. As shown in figure 7, the stress triaxiality decreases with the increase of load frequency, and the stress triaxiality basically remains 
unchanged with the increase of load amplitude.

The reason for this may be that the brain-like soft material itself has approximately incompressible properties, with a bulk modulus of $2.19 \mathrm{GPa}$, but relatively weaker shear resistance, with the steady-state shear modulus of 2161.4 Pa. Therefore, although the stress amplitude of the secondary shear wave is small, it can also cause a large shear strain in the softbrain-like material domain and cause damage to the soft-brain-like material.
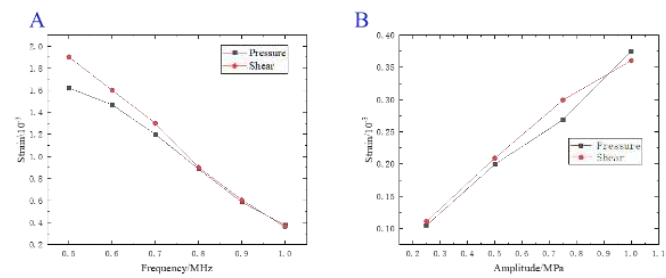

Figure 6: Shear and bulk strain amplitude under different frequency and different amplitude load: A. Obtained at $1 \mathrm{MPa}$ amplitude load; B. Obtained at $1 \mathrm{MHz}$ frequency load
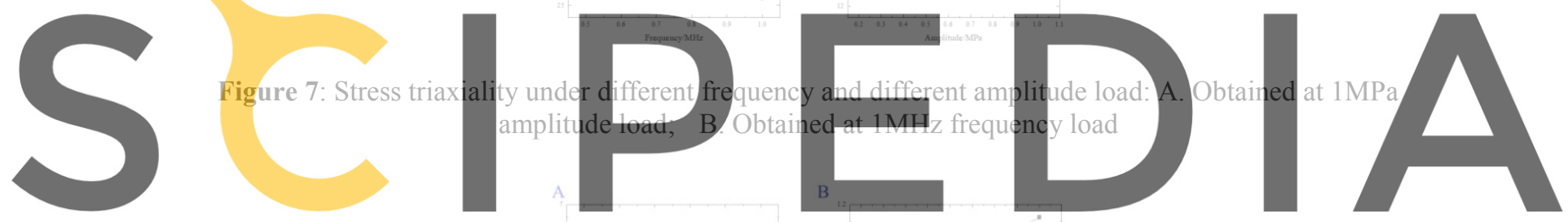

Register for free at https//www.scipedia.com to download the version without the watermark

Figure 8: Regularized distortional wave component under different frequency and different amplitude load: A. Obtained at $1 \mathrm{MPa}$ amplitude load; B. Obtained at $1 \mathrm{MHz}$ frequency load

Referring to the method of magnetic resonance elastography ${ }^{[20]}$, introduce the distortional wave component normal to the image plane:

$$
\Gamma=\left(\partial u_{2} / \partial x_{1}-\partial u_{1} / \partial x_{2}\right) / 2
$$

The root-mean-square value of the distortion $\Gamma$ was computed and normalized by the rootmean-squared applied pressure load according to the following expression:

$$
\Gamma / P=\sqrt{(1 / T) \int_{0}^{T}[\Gamma(t)]^{2} d t} / \sqrt{(1 / T) \int_{0}^{T}[P(t)]^{2} d t}
$$

As shown in figure 8, using this index to analyze the shear wave in brain-like soft material, 
it can be found that the distortion wave component decreases gradually with the increase of load frequency and increases gradually with the increase of load amplitude, which can explain that with the decrease of load frequency and the increase of load amplitude, more distortion can enter the focus area.

\subsection{Energy dissipation characteristics of the feature point and focus area}

This section mainly analyzes the energy dissipation of brain-like soft material in the process of ultrasonic focusing. The energy dissipation is mainly related to the viscoelastic behavior of materials. In this paper, the approximate incompressibility of brain-like soft material is taken into account when the constitutive model is established, so it is considered that there is no viscoelastic effect in volume deformation and only viscoelastic effect in shear deformation.

The shear stress and strain of the characteristic points in the focus area can be observed first. It can be found that the stress-strain curve forms a series of hysteresis loops, and the dissipation energy can be obtained by integrating the area around the hysteresis loop. Taking a single hysteresis loop for analysis, the corresponding dynamic shear modulus can be obtained [21].

The focus model is loaded with a $1 \mathrm{MHz}$ load with a different amplitude $(0.25-1 \mathrm{MPa})$, and the effect on the focus effect is observed. As shown in figure 9, the shear hysteresis curves of the focus area under different amplitude loads are drawn. On the hand, it can be found that the area of the hysteresis loop decreases gradually with the increase of frequency, that is, the speed of corresponding dissipation energy decreases; the ratio of the long axis and the short axis of the ellipse corresponding to the hysteresis loop is getting smaller and smaller, that is, the $\tan \&$ is unchanged. On the o corresponding to different amplitudes are the hysteresis curve gradually decreases, whic affect the magnitude of the dynatnic modulus.

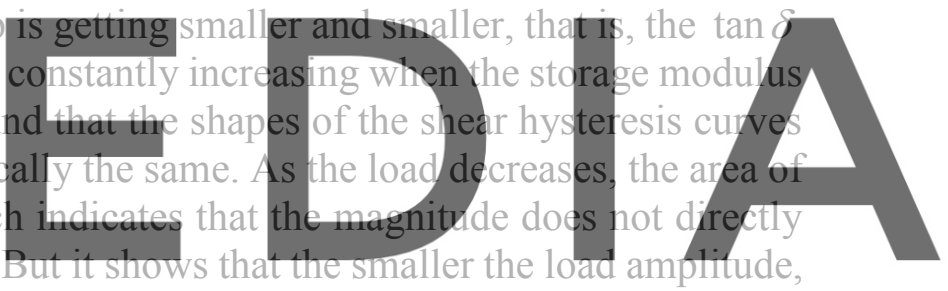
the smaller the energy dissipated by a single hysteresis loop. And the frequency does not change,

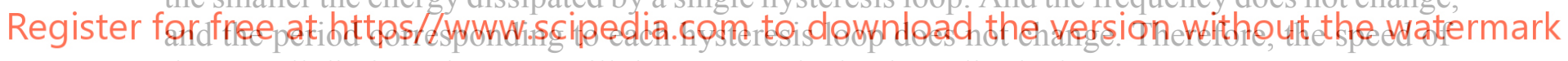
the overall dissipated energy will decrease as the load amplitude decreases.

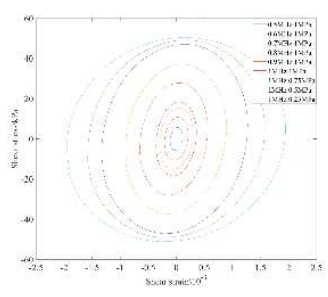

Figure 9: Shear hysteretic curves under loads of different frequencies and amplitudes

The above analysis for the hysteresis loop mainly reflects the information of the feature point in the focus area under the action of the ultrasonic signal, and mainly reflects the characteristics of the constitutive equation of brain-like soft material. In order to further consider the effect of focusing on the energy attenuation, we analyzed the attenuation coefficient of ultrasound, the lateral resolution of focused ultrasound and the temperature rise rate directly related to the energy attenuation. 
Futhermore, in order to obtain the attenuation coefficient of ultrasound, it can be simplified on the original model. The load is directly applied to the lower end surface of the cylindrical biological tissue, and the same loading mode can be used to obtain the attenuation of the ultrasonic plane wave in the biological tissue along with the incident depth, which is used to fit the corresponding attenuation coefficient:

$$
\alpha=\log \left(A_{0} / A\right) / d
$$

where $A_{0}$ is the amplitude of the reference pressure, and $A$ is the pressure amplitude of the corresponding position, and $d$ is the distance of the corresponding position from the reference position.

As shown by the blue curve in figure 10A, as the frequency increases, the attenuation coefficient gradually increases. According to the empirical formula $\alpha=a f^{b}$, the relationship between the attenuation coefficient and frequency can be fitted, and the parameters a $=0.06861 \mathrm{~mm}^{-1} \mathrm{MHz}^{-\mathrm{b}}, \mathrm{b}=1.977 \mathrm{can}$ be obtained. The rising trend of the attenuation coefficient is consistent with the rising trend of the loss modulus obtained from the hysteresis image. It means that as the frequency increases, the attenuation of the ultrasonic amplitude will be faster, which means that the amplitude will be smaller when the ultrasonic waves reach the focused area.
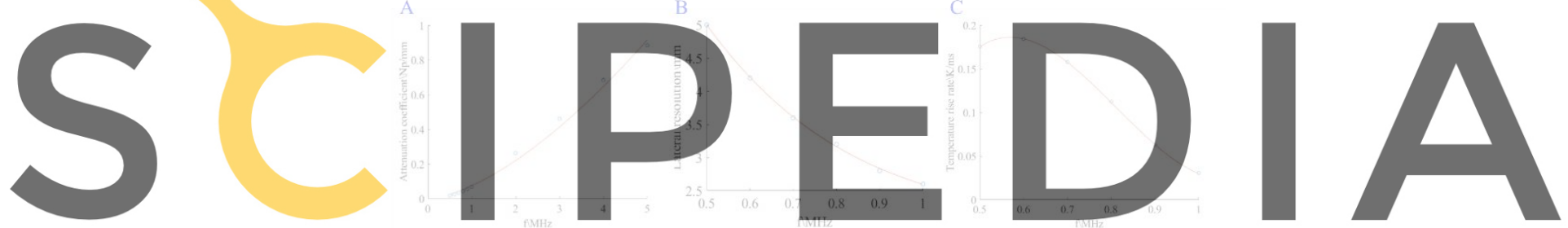

Figure 10: A. Shear hysteretic curves under loads of different frequencies and amplitudes; B. The relationship

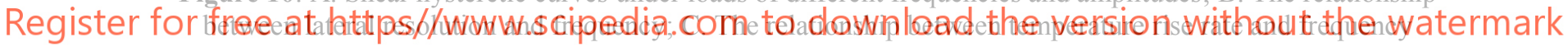

Further, in order to study the energy input of the focused area, the longitudinal resolution of the focused ultrasound can be taken as the amount of investigation. As shown by the green curve in figure $10 \mathrm{~B}$, as the frequency increases, the lateral resolution of the focused ultrasound increases, indicating that the degree of energy accumulation will increase as the frequency increases.

In terms of the attenuation coefficient and lateral resolution, the higher the frequency, the lower the amplitude of the ultrasonic wave in the focused area, but the energy will be more concentrated. There is a contradiction between the two and it will affect the energy attenuation in the focus area at the same time. Therefore, a direct indicator of the temperature rise effect is needed to judge the true dissipation situation. Use the least square fitting method to fit the timetemperature curve to get the temperature rise rate, forming the result of the red curve in figure $10 \mathrm{C}$. Finally, it can be found that there is a maximum value of the temperature rise coefficient in $0.5-0.6 \mathrm{MHz}$.

This result can also be used in ultrasound imaging and focused ultrasound ablation. In order to get better echo quality, the ultrasonic amplitude and the longitudinal resolution should be as small as possible, and the result should be consistent with the extreme value of the 
temperature rise rate. In the focused ultrasound ablation, in order to avoid damaging the biological tissue in the non-target position as far as possible, it is necessary to focus on high precision and fast temperature rise speed, which is consistent with the frequency required by the above maximum temperature rise rate.

\section{CONCLUSIONS}

Focused ultrasound is widely used in medical diagnostic imaging, non-invasive treatment, and other fields, but the research on the quantification of its physical effects and the corresponding biological effects is not in-depth enough. In this paper, the interaction process between focused ultrasound and brain-like soft material is abstractly simplified, and an acoustic-solid-thermal coupling model is established to describe the interaction between focused ultrasound and brain-like soft material. In this paper, the dynamic mechanical behavior of brain-like soft material is described using a hyper-viscoelastic constitutive model, and it is used in combination with the model to process the focused ultrasound and soft-brain-like tissue under different frequencies and amplitude loads. The following conclusions were reached:

- $\quad$ According to the stress-strain response, brain-like soft materials have the characteristics of high compression resistance and low shear resistance. Under the action of focused ultrasound, the magnitudes of shear strain and volume strain are the same, but the volume stress is two or three orders of magnitude larger than the shear stress.

- $\quad$ Under the action of focused ultrasound, the dynamic mechanical response of brainlike soft material will change with the change of load. With the increase of load frequency, the energy input to the focus region is smaller, but the loss ratio will increase. With the increase of load amplitude, the more energy is input into the focus region, the more energy is lost.

- With the increase of the load frequency of focused ultrasound, the attenuation coefficient of ultrasound increases gradually, which has a specific exponential relationship with the ultrasonic frequency; the width of the focused area decreases with the increase of frequency, and the lateral resolution of focused ultrasound increases gradually. To obtain higher imaging quality or higher temperature rise, the optimal solution can be found in a specific frequency range.

In this paper, the law of the interaction between focused ultrasound and brain-like soft material is simulated, and the method of obtaining dynamic modulus by using the law of stress and strain in the focus area of ultrasound is obtained, which provides the basis for the application of focused ultrasound in elastic imaging and viscoelastic property inversion of materials. Meanwhile, the focusing characteristics of ultrasound and the dynamic mechanical response of brain-like soft material under different frequency and amplitude loads are obtained, which provides support for the application and improvement of focused ultrasound in medical diagnosis and treatment.

\section{REFERENCES}

[1] Shung K K. Diagnostic ultrasound: Imaging and blood flow measurements. CRC press, 2015. 
[2] Ter Haar G. Therapeutic applications of ultrasound. Progress in biophysics and molecular biology (2007) 93(1-3): 111-129.

[3] Park J, Zhang Y, Vykhodtseva N, et al. The kinetics of blood brain barrier permeability and targeted doxorubicin delivery into brain induced by focused ultrasound. Journal of controlled release (2012) 162(1): 134-142.

[4] Samiotaki G, Vlachos F, Tung Y S, et al. A quantitative pressure and microbubble - size dependence study of focused ultrasound - induced blood - brain barrier opening reversibility in vivo using MRI[J]. Magnetic resonance in medicine (2012) 67(3): 769-777.

[5] Pervin F, Chen W W. Dynamic mechanical response of bovine gray matter and white matter brain tissues under compression. Journal of biomechanics (2009) 42(6): 731-735.

[6] Song B, Chen W. Dynamic stress equilibration in split Hopkinson pressure bar tests on soft materials. Experimental mechanics (2004) 44(3): 300-312.

[7] Chen W, Zhang B, Forrestal M J. A split Hopkinson bar technique for low-impedance materials. Experimental mechanics (1999) 39(2): 81-85.

[8] Song B, Ge Y, Chen W W, et al. Radial inertia effects in Kolsky bar testing of extra-soft specimens. Experimental Mechanics (2007) 47(5): 659.

[9] Song B, Chen W, Ge Y, et al. Dynamic and quasi-static compressive response of porcine muscle. Journal of biomechanics (2007) 40(13): 2999-3005.

[10] Huang J, Holt R G, Cleveland R O, et al. Experimental validation of a tractable numerical model for focused ultrasound heating in flow-through tissue phantoms. The Journal of the Acoustical Society of America (2004) 116(4): 2451-2458.

[11] Chafi M S, Ganpule S, Gu L, et al. Dynamic response of brain subjected to blast loadings: influence of frequency ranges. International Journal of Applied Mechanics (2011) 3(04): 803-823.

[12] Bilston L E, Liu Z, Phan-Thien N. Linear viscoelastic properties of bovine brain tissue in shear. Biorheology (1997) 34(6): 377-385.

[13] Nicolle S, Lounis M, Willinger R, et al. Shear linear behavior of brain tissue over a large frequency range. Biorheology (2005) 42(3): 209-223.

[14] Brands D W A. The large shear strain dynamic behavior of in-vitro porcine brain tissue and a silicone gel model material. SAE Technical Paper (2000).

[15] Arbogast K B, Margulies S S. Regional differences in mechanical properties of the porcine central nervous system. SAE transactions (1997): 3807-3814.

[16] Arbogast K B, Margulies S S. Material characterization of the brainstem from oscillatory shear tests[J]. Journal of biomechanics (1998) 31(9): 801-807.

[17] Lippert S A, Rang E M, Grimm M J. The high frequency properties of brain tissue. Biorheology (2004) 41(6): 681-691.

[18] Shuck L Z, Advani S H. Rheological response of human brain tissue in shear. (1972).

[19] Moore D F, Jérusalem A, Nyein M, et al. Computational biology—modeling of primary blast effects on the central nervous system. Neuroimage (2009) 47: T10-T20.

[20] Lakes R, Lakes R S. Viscoelastic materials[M]. Cambridge university press (2009).

[21] Clayton E H, Genin G M , Bayly P V . Transmission, attenuation and reflection of shear waves in the human brain. Journal of The Royal Society Interface (2012) 9(76):2899-2910. 